\title{
Nanotechnology in the targeted drug delivery for bone diseases and bone regeneration
}

This article was published in the following Dove Press journal:

International Journal of Nanomedicine

24 June 2013

Number of times this article has been viewed

\section{Wenyi Gu ${ }^{1,2}$ \\ Chengtie $\mathrm{Wu}^{3}$ \\ Jiezhong Chen' \\ Yin Xiao'}

'Institute of Health and Biomedical Innovation, Queensland University of Technology, Brisbane, QLD, Australia; ${ }^{2}$ Australian Institute for Bioengineering and Nanotechnology, The University of Queensland, Brisbane, QLD, Australia; ${ }^{3}$ State Key Laboratory of High Performance Ceramics and Superfine Microstructure, Shanghai Institute of Ceramics, Chinese Academy of Sciences, Shanghai, People's Republic of China
Correspondence: Yin Xiao Institute of Health and Biomedical Innovation, Queensland University of Technology, 60 Musk Avenue, Kelvin Grove, Brisbane, QLD, 4059, Australia

Tel +6 I 731386240

Fax +6I 731386030

Email yin.xiao@qut.edu.au
Abstract: Nanotechnology is a vigorous research area and one of its important applications is in biomedical sciences. Among biomedical applications, targeted drug delivery is one of the most extensively studied subjects. Nanostructured particles and scaffolds have been widely studied for increasing treatment efficacy and specificity of present treatment approaches. Similarly, this technique has been used for treating bone diseases including bone regeneration. In this review, we have summarized and highlighted the recent advancement of nanostructured particles and scaffolds for the treatment of cancer bone metastasis, osteosarcoma, bone infections and inflammatory diseases, osteoarthritis, as well as for bone regeneration. Nanoparticles used to deliver deoxyribonucleic acid and ribonucleic acid molecules to specific bone sites for gene therapies are also included. The investigation of the implications of nanoparticles in bone diseases have just begun, and has already shown some promising potential. Further studies have to be conducted, aimed specifically at assessing targeted delivery and bioactive scaffolds to further improve their efficacy before they can be used clinically.

Keywords: nanoparticles, nanostructured scaffold, cancer bone metastasis, bone diseases, target drug delivery, bone regeneration

\section{Introduction}

Nanotechnology has changed our daily lives in many ways, including in matters related to energy, the environment, and medicine. With respect to medicine, nanomaterials offer new tools to explore diseases using imaging and diagnostic applications, ${ }^{1}$ and more popularly, they act as vehicles for delivering drugs or therapeutic agents to achieve better and safer treatment outcomes. ${ }^{2-6}$ In addition, nanomaterials can provide a fine structure (scaffold) for tissue regeneration, which is currently revolutionizing tissue engineering in medicine. ${ }^{7}$

Bone diseases represent a variety of skeletal-related disorders including defects that cause major mobility hindrance and mortality to human beings. As no effective treatments are available for some of the most common skeleton disorders such as arthritis, osteoarthritis, osteosarcoma, and metastatic bone cancer, there is an urgent need to develop new drugs and drug delivery systems for safe and efficient clinical treatments. In the development of treatments for bone degenerative diseases (osteoarthritis) and bone cancers, the balance between medication side effects and treatment efficacy is always an issue. To address these issues and to increase the efficacy of treatment, a targeted delivery using nanotechnology has been widely proposed as a potential strategy. 
Targeted delivery is an important goal to achieve for nanomedicine. Nanoparticles (NPs) can offer many unique features for the potential targeted delivery of treatments for bone diseases. The advantages of using NPs may include: (1) carrying the drug to its destination while keeping the drug concentrated so that once endocytosed by cells, the drug can maximize its effect; (2) protecting the drug from being dispersed or degraded by body fluids and increasing the circulation time or retention time in the body; (3) carrying more drug molecules and increasing the solubility of some hydrophobic drugs due to the large surface area of NPs; and (4) loading other targeting molecules to achieve specific delivery via surface modification of NPs.

The types of NPs used for the investigation of drug delivery for bone diseases can be classified into organic and inorganic NPs. Organic NPs typically include poly-L-lysineand polymer-based (eg, poly[lactic-co-glycolic acid] PLGA) NPs, $, 5,8$ and inorganic NPs mainly include silica-based mesoporous NPs and layered double hydroxides (LDH) ${ }^{10,11}$ The drugs delivered for bone diseases include traditionally used drugs such as antibiotics and chemotherapeutics, and gene therapy reagents like plasmid deoxyribonucleic acid (DNA) or small interfering ribonucleic acid (siRNA). Currently, targeted delivery is mainly achieved by using special drugs called bisphosphonates (BPs), which have been used for treating bone diseases ${ }^{12-16}$ they are also bone affinity agents that are grafted onto NPs for the specific delivery of other drugs to bone tissues. ${ }^{17}$ Although there is no nanodelivered treatment available in clinic so far, better designed NPs with safe (low or no toxicity) and multifunctional properties will offer specificity for the treatment of bone diseases. For example, multifunctional NPs have been recently developed from the combination of organic and inorganic NPs, ${ }^{18-22}$ which may eventually be applied to bone diseases. Some more finelytuned nanomaterials have also been studied in bone regeneration because these nanostructured scaffolds can offer some new functions for the controlled release of growth factors or cytokines to promote and regulate the surrounding cells for new bone formation.
In this review, we summarized the most recent progress of NPs in studies of bone diseases including cancer metastasized into bone, osteoarthritis, osteosarcoma, bone infections, inflammatory diseases, and bone regeneration. We raise some issues (including safety issues) and concerns about the current methods and hope these discussions will provide future directions surrounding nanotechnology and its application for bone-related diseases and bone regeneration.

\section{Targeted delivery for preventing and treating cancer bone metastasis}

Tumor metastasis is a major cause of cancer-related deaths, and the bone tissue is a major site for several cancers to metastasize. ${ }^{23}$ Particularly, bone is the only site of metastasis for prostate cancer, whereas approximately $70 \%$ of metastatic breast cancers metastasize to bone. ${ }^{23,24}$ Therefore, the treatment of metastasized cancers in bone (termed "cancer bone metastasis" hereafter) is important for patients in providing prolonged survival rates; it has also recently emerged as an important bone disease to target. As described by Coleman, ${ }^{25}$ the skeleton is the most common organ to be affected by metastatic cancer, and this is the site of the disease that produces the greatest morbidity rates. This statement further emphasises the demand and importance of developing safe, effective, and targeted nonviral drug or gene carriers for the clinical success of treating bone diseases including bone metastatic cancers.

When treating cancer bone metastasis, one particular class of drug that has attracted lots of attention is BPs. BPs are well established and are commonly used for the treatment of bone diseases due to their specific affinity to bone tissues. This property makes BPs particularly useful, as it delivers NPs to bone tissue. The known functions of BPs include that they can strengthen bone, treat or prevent osteoporosis, and treat Paget's disease of bone. ${ }^{26-28}$ However, the emerging data suggest that BPs also have antitumor properties and can be used to treat cancer bone metastases. ${ }^{12,29,30}$ BPs have undergone three generations (Table 1). Early clinical data on the prevention of bone metastases by the early-generation

Table I Anticancer effect of BPs/nanoparticles/anticancer agent complex

\begin{tabular}{llllll}
\hline Generation & Drug & Nanoparticles bound & Anticancer agents & Effects & References \\
\hline I & Bp clodronate & Liposomes & Clodronate & Inhibition of cell growth & Decreased metastasis \\
2 & Zoledronic acid & PLGA & Docetaxel & $\begin{array}{l}\text { Increased cellular uptake } \\
\text { Prolonged half-life }\end{array}$ \\
3 & Risedronate & PLL-CD & Cyclodextrin & Prevention of bone metastasis & 9
\end{tabular}

Abbreviations: BP, bisphosphonate; PLGA, poly lactic-co-glycolic acid; PLL-CD, poly-L-lysine covalented beta-cyclodextrin. 
$\mathrm{BP}$, clodronate, have yielded promising results in patients with breast cancer, and trials have been undertaken to assess its efficacy. ${ }^{31,32}$ However, recent data indicate that this BP may only be effective in older women who are no longer undergoing menopause..$^{33}$ Similarly, the new generation BP, zoledronic acid, has demonstrated activity in the prevention of bone metastases. In a 5-year trial, the overall survival rate of patients with multiple myeloma was greater in patients whose standard treatment regimens included zoledronic acid compared with standard treatment alone $(P<0.01){ }^{15}$

In other preclinical studies, it has been demonstrated that the second-generation BPs (zoledronic acid) can inhibit angiogenesis, invasion and adhesion of tumor cells, and overall tumor progression, and emerging evidence suggests that the use of these agents may block the development of bone metastases. ${ }^{13}$ In clinical studies of patients with cancer bone metastasis, serum levels of vascular endothelial grouth factor (VEGF), an vital factor for angiogenesis, were significantly reduced in patients receiving zoledronic acid, suggesting that zoledronic acid may have a property of inhibiting angiogenesis. ${ }^{27,34,35}$ Now, third-generation BPs (risedronate [RIS]) are available, and they are believed to be more effective and result in less toxicity. However, a recent study showed that using RIS as an additive drug of docetaxel did not have a better treatment effect on prostate cancer patients, ${ }^{36}$ suggesting that BPs alone have limited treatment effects on cancer patients in clinical settings.

NPs have been developed to deliver BPs to increase the efficacy of the drug (Table 1). For example, Daubiné et al ${ }^{9}$ employed poly-1-lysine covalently grafted with beta-cyclodextrin as a polycationic vector (PLL-CD) for RIS delivery. The authors showed that the efficacy of RIS at inhibiting cancer cell invasion in vitro was strongly enhanced upon complexation, irrespective of whether PLL-CD:RIS complexes were in solution status or embedded into polyelectrolyte multilayered nanoarchitectures. It has also been demonstrated in vivo that complexes in solution status clearly prevented cancer-induced bone metastasis in animal models. ${ }^{9}$

NPs have also been reported to deliver second-generation BPs (zoledronate [ZOL]) to increase their efficacy. As ZOL has a strong affinity towards bone tissue, it has been used to deliver docetaxel into bone and showed significant synergism in the treatment of bone metastasis. ${ }^{38}$ It has been demonstrated that ZOL-conjugated PLGA NPs exhibit greater cellular uptake than pegylated PLGA NPs, with changes in the cellular uptake route. In vitro studies on the breast cancer cell lines of MCF-7 and BO2, as well as ZOL-anchored PLGAPEG NPs, have shown enhanced cell cytotoxicity, increased in-cell cycle arrest, and more apoptotic activity. In animal studies, the technetium-99 m radio-labeling ZOL-tagged NPs also exhibited a prolong blood circulation half-life, reduced liver uptake, and significantly higher retention at the bone site with enhanced tumor retention. ${ }^{38}$

Since BPs have a special affinity to bone, they are used as targeting molecules on NPs to deliver other anticancer drugs (Figure 1). Salerno et $\mathrm{al}^{39}$ reported biodegradable, biocompatible NPs made of a conjugate between poly (D, L-lactide-co-glycolic) acid and alendronate (ALE), which are suitable for systemic administration, and for directly targeting the site of tumor-induced osteolysis. Specifically, the NPs were used to load doxorubicin (DXR) and were evaluated for their antitumor effects in primary or metastatic bone tumors in an orthotopic mouse model of breast cancer bone metastases. The results showed that in vitro, both free DXR and DXR-loaded NPs exhibited a significant dose-dependent growth inhibition of the breast cancer cells. Similarly, both DXR-loaded NPs and free DXR reduced the incidence of metastases in mice though the advantage of the NP loaded drug was not clearly demonstrated in this study. ${ }^{39} \mathrm{NP}$ loading of the drug was not clearly demonstrated in this study.

Recently, a novel bone-seeking polymer NP was reported. In this system, an amino-BP, ALE was bound covalently to a biodegradable polymer, PLGA, containing a free-end carboxylic group. Blood compatibility and cytotoxicity of the NPs were assessed in vitro. Owing to the presence of the BP residue, PLGA-ALE NPs were adsorbed onto hydroxyapatite

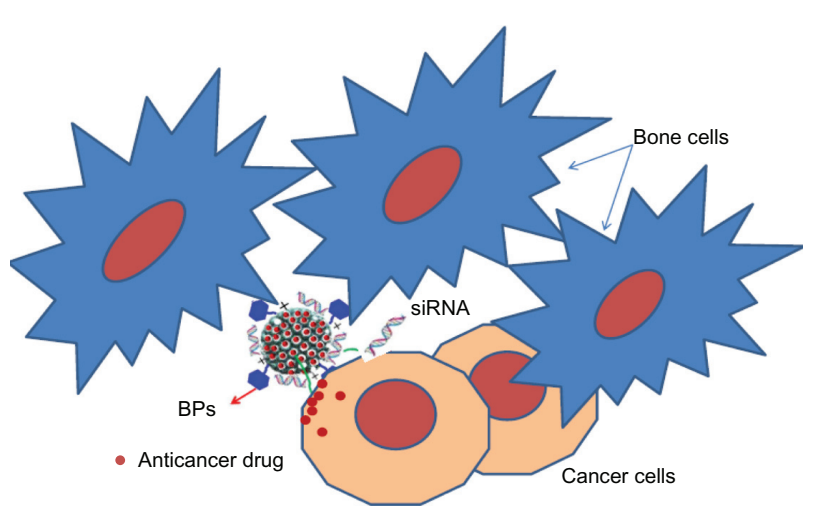

Figure I Multifunctional NPs for targeted delivery to bone metastasized cancer cells.

Notes: A schematic figure shows the use of a multifunctional NP to deliver anticancer reagents and gene therapy (eg, siRNA). In this scheme, the NP of a mesoporous silica NP is loaded with anticancer drugs in the pore, and the NP surface is grafted with BPs or other bone-specific markers to target bone cells or tissues. Because of the positive charge of the NP surface, it can also carry negatively charged siRNA for gene therapy. When the NP is administered, it will specifically remain at the sites of bone cells where/with which the metastasized cancer cells are closely associated. The cancer cells will be killed by the released drugs or siRNAs.

Abbreviations: NP, nanoparticle; BP, bisphosphonate; siRNA, small interfering ribonucleic acid. 
(HAp) to a higher extent than pure PLGA NPs. The PLGAALE conjugate did not induce hemolysis, alterations of the plasmatic phase of coagulation, or cytotoxic effects on endothelial cells and trabecular osteoblasts. The authors believe that this conjugate is a novel biomaterial that is able to provide NPs, which can be further loaded with drugs, such as anticancer agents, and used for osteolytic or other bone diseases. ${ }^{17} \mathrm{~A}$ similar delivery system was also reported by Pillai et $a 1,{ }^{40}$ in which the researchers showed that PLGA NPs can effectively deliver antibiotics (such as nafcillin) to osteoblasts and kill intracellular bacteria $S$. aureus in these cells.

Another study using a similar strategy showed that a direct conjugate PTX-PEG-ALN NP exhibited an improved pharmacokinetic profile when compared with the free drugs due to the marked increase in their half-life. ${ }^{41}$ In this NP, PTX is a potent anticancer drug that can result in severe side effects, originating from both the drug itself and its solubilizing formulation, Cremophor $^{\circledR}$ EL. ${ }^{41}$ ALN is an aminobisphosphonate used for the treatment of osteoporosis and bone metastases, as well as for bone targeting. This conjugate was demonstrated to have a great binding affinity to the bone mineral HAp in vitro, and an IC (50) comparable to that of the combination of free drugs in the cells of human adenocarcinoma of the prostate (PC3). ${ }^{41}$ In addition, PTX-PEG-ALN could be solubilized directly in physiological solutions without the need for Cremophor ${ }^{\circledR}$ EL. The data presented here encourage further investigations on the potential of PTX-PEG-ALN as a treatment for cancer bone metastases.

All of the above data indicate that the combination of the specificity of treatment effects of bone affinity BPs with the efficiency of PLGA delivery is one of the optimal strategies for the future development of effective treatments of bone metastasis. Some positive results have also been obtained in in vitro studies, which promise further optimizations of these systems for in vivo or preclinical studies. BPs and DXR are not the only drugs used to treat bone metastasis; other chemotherapeutic drugs, such as cisplatin, can also be used. NP deliveries of these drugs have been demonstrated to increase treatment efficacy in vitro; ${ }^{42,43}$ however, in vivo model testing is still necessary to validate these delivery systems.

In addition to the above advance in targeted drug delivery, a deep understanding of the possible mechanisms of why and how cancer cells migrate specifically to bone sites would be an important direction of research for developing targeted treatment to bone metastasis. So far, the reason why cancer cells metastasize to bone sites or tissues is not very clear, although there is evidence showing that bone sialoprotein and osteopontin are important factors in the metastasis of breast cancer. ${ }^{44}$ A recent study showed that silencing these genes with specific small interfering RNA (siRNA) or antisense could inhibit metastasis of breast cancer in a nude rat model. ${ }^{8,44}$ These studies suggest that targeting bone tissue biomarkers could be another strategy to stop cancer bone metastasis. Therefore, combining delivery systems of NPs, selective drugs, and gene therapy may be a new research direction to develop more effective treatment and prevention for bone metastasis (Figure 1).

It is generally believed that the enriched nutrients and the relatively stable environment are important factors for tumor cell migration to the bone tissue and subsequent growth. However, questions remain as to what kind of cancer cells are responsible for this migration, and how the interaction between these migrating cancer cells and bone cells occur. Therefore, better understanding of cancer cells (especially cancer stem cells) and their migration properties may help us to identify potential therapeutic targets and develop targeted nanodelivery to prevent or inhibit bone metastasis.

\section{Osteosarcoma}

Although osteosarcoma needs more effective and safe treatments rather than the conventional therapies, such as chemotherapy, radiotherapy and surgery, the application of nanotechnology for targeted delivery in the treatment of osteosarcoma is not prevalent. Actually, only limited numbers of studies have been carried out on this subject. A study carried by Federman et $\mathrm{al}^{45}$ reported an osteosarcoma-associated cell surface antigen, ALCAM and engineered anti-ALCAMhybrid polymerized liposomal NP immunoconjugate, alpha-AL-HPLN. The authors used this NP to specifically deliver chemotherapy drug DXR to osteosarcoma cells and showed that an anti-ALCAM-hybrid polymerized liposomal nanoparticle (alpha-AL-HPLN) had significantly enhanced cytotoxicity over untargeted hybrid polymerized liposomal nanoparticles, and over a conventional liposomal DXR formulation. ${ }^{45}$ Besides, magnetic arsenic trioxide NPs were shown to have targeted effects on osteosarcoma cells by applying a magnetic field, ${ }^{46}$ while calcium phosphate NPs were shown to be able to deliver the anticancer drug, cisplatin, and exhibit cytotoxic effects to a murine osteosarcoma cell line (K8) in a dose-dependent manner. ${ }^{43}$

For the general treatment of osteosarcoma, Susa et $\mathrm{al}^{42}$ reported biocompatible, lipid-modified, dextran-based polymeric NPs and showed that the NPs loaded with DXR had a curative effect on multidrug resistant osteosarcoma cells by increasing the amount of drug accumulation in the nucleus, and increased apoptosis in osteosarcoma cells as 
compared with DXR alone. Similarly, Sun et $\mathrm{al}^{47}$ showed a combined strategy of chemotherapy and gene therapy in a single dextran-polyethylenimine (PEI)-NP. Both DXR and PEI were grafted to a dextran chain, and plasmid DNA could also be loaded, as PEI can provide the positive charge to load the negative charged plasmid. When the NPs were loaded with DXR, they showed a higher cytotoxicity compared to free DXR in MG-63 and Saos-2 osteosarcoma cells though DEX-PEI, which maintained over $65 \%$ cell viability at a concentration of $8 \mathrm{mg} / \mathrm{mL}$. The authors also demonstrated that the NPs can efficiently deliver plasmid pEGFP-N1 into osteosarcoma cells with low cytotoxicity. This system can be useful for delivering both chemotherapy and gene therapy for osteosarcoma.

Besides the above mentioned NPs and delivery systems, quite a few numbers of reports related to OS have focused on developing nanodelivery systems, and osteosarcoma cells were used as a testing model. For instance, various types of NPs including mesoporous silica NPs, ${ }^{10}$ gold NPs, ${ }^{48}$ PEI-coated gold NPs,${ }^{49}$ polymeric NPs, ${ }^{50-52}$ quantum dots, ${ }^{53}$ liposomes,${ }^{54}$ and LDH have been tested for delivering anticancer drugs or siRNA in osteosarcoma or Ewing's sarcoma cell lines. ${ }^{11}$ Furthermore, chitosan (CS) NPs were reported to encapsulate DNA enzyme, Dz13, to effectively inhibit osteosarcoma growth. ${ }^{55}$ PLGA NPs were also employed to deliver the chemotherapy drug, cisplatin, for the treatment of osteosarcomas. ${ }^{56}$ Gelatin A and B were used to synthesize NPs, and cell uptake in osteosarcoma cells was tested in order to develop a delivery system for osteosarcomas. ${ }^{57}$ Even though they were used as a model, these NPs could be potentially used for future application in developing treatments for osteosarcomas. We believe that more targeted delivery and therapeutic approaches with NPs loaded with bone-specific affinity reagents such as BPs will be expected in the near future. There are some studies exploring NPs for the delivery of gene therapy in osteosarcoma treatment, and we will summarize them later, together with gene therapies for other bone diseases.

\section{Osteoarthritis}

NPs have been explored in terms of their application to deliver drugs for osteoarthritis treatment, in the sense that NPs could be useful as a local delivery system for osteoarthritis drugs, and this could increase the drug retention time in local tissues or fluids (please see more in Figure 2). For example, cationic polymeric hydrogel was reported to increase the retention time of a model drug, dextran, after ionically cross-linked with the NP in synovial fluid without influence on the feature of the fluid. ${ }^{58}$ Self-assembling copolymer

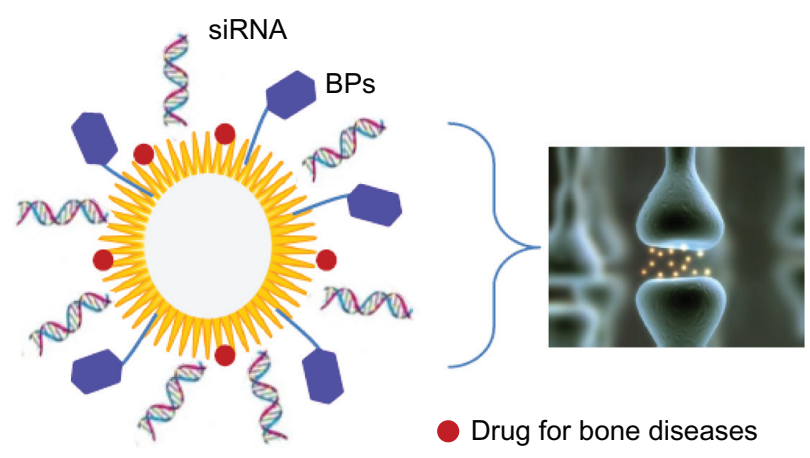

Figure 2 Biodegradable (polymer-based) NP-targeted local delivery in bone. Notes: A schematic diagram shows an ideal NP that can load multiple cargos, including targeting molecules that have an affinity to bone tissue or cells such as BPs, siRNA for gene therapy, and drugs for bone diseases. These kinds of NPs are better and readily biodegradable, such as polymer-based NPs or some kinds of inorganic NPs like layered double hydroxides. They can be further modified to be multifunctional NP carriers, but will not be silica based particles to increase the roughness of the interaction surface of joints for local delivery.

Abbreviations: NP, nanoparticle; siRNA, small interfering ribonucleic acid; $\mathrm{BP}$, bisphosphonate.

NPs were also shown to increase the retention time of IL-1 receptor antagonist (IL-1Ra), the natural protein inhibitor of IL-1, when it was covalently conjugated on the surface of the NPs and delivered locally. ${ }^{59}$ In addition, a CS vector was used to deliver gene therapy, and DNA sequences encoding $I L-1 R a$ and $I L-10$ were delivered and shown to be effective in inhibiting the development of osteoarthritis in a rabbit model. ${ }^{60}$ Apart from drug delivery, NPs are also studied in arthritis imaging; for example, ultrasmall superparamagnetic iron oxides were used for magnetic resonance imaging in rat arthritis model. ${ }^{61}$ In another study, gold NPs were shown to be coupled with a fiber-optic particle plasmon resonance technique to sense the IL-1-beta level in synovial fluids, which has a diagnostic value for osteoarthritis. ${ }^{62}$ Nanofiber was also used to make nanofibrous scaffolds for engineered meniscus construction to increase cell survival. ${ }^{63}$

\section{Infection and inflammatory bone diseases}

In the past 10 years, a lot of efforts have been made to develop nanodelivery systems for treating bone-related diseases. NPs have been shown to have some advantages for delivering conventional drugs such as antibiotics and other antiinflammatory drugs. A review article from 2009 has summarized most of the advances of nanotechnology for drug delivery in bone-related diseases. ${ }^{64}$ Here, we summarized the updates from the last 2-3 years.

NPs are considered to be a prospective candidate in achieving high local bioactivity and low systemic side effects of antibiotics in the treatment of dental, periodontal, and bone infections 
for localized and temporally controlled delivery. Feng et $\mathrm{al}^{65}$ reported a three-dimensional (3D) porous tissue engineering scaffold, which was able to release antibiotics in a controlled fashion for long-term inhibition of bacterial growth. A highly soluble antibiotic, doxycycline (DOXY), was incorporated into PLGA nanospheres (NSs) using a water-in-oil-in-oil (w/o/o) emulsion method. The PLGA NSs were then incorporated into prefabricated nanofibrous PLGA scaffolds with a well interconnected macroporous structure. The study of release kinetics of DOXY showed that DOXY release from the NS-scaffolds occurred in a locally and temporally controlled manner. In vitro antibacterial tests of such scaffolds loaded with DOXY showed its ability to inhibit common bacterial growth (S. aureus and E. coli) for a prolonged duration. The successful incorporation of DOXY onto 3D scaffolds and its controlled release from scaffolds suggests that the usage of nanofibrous scaffolds in the delivery of large molecules (such as growth factors) to the delivery of small hydrophilic drugs allows for a broader application and more complex tissue engineering strategy. ${ }^{65}$ Similarly, PLGA was employed to compose a poly (ethylene glycol) monomethyl ether (mPEG) and PLGA copolymer as a sol-gel drug delivery system for treating osteomyelitis. ${ }^{66}$ This delivery system was shown to have several advantages in treating osteomyelitis, including easy preparation, $100 \%$ encapsulation rate, near-linear sustained release of drugs, injectable design, and in situ gelling of the target tissue.$^{66}$ In addition, it was shown in the study that similar to the undegradable teicoplanin-impregnated polymethylmethacrylate bone cements, the implantation of the mPEG-PLGA hydrogel-containing teicoplanin was effective for treating osteomyelitis in a rabbit model as evidenced by histological examination and immunoblotting analyses. These data suggest that the use of the mPEG-PLGA-based biodegradable hydrogels may hold great promise as a therapeutic strategy for other bone infections. ${ }^{66}$

Apart from PLGA-related nanomaterials, other targeted delivery systems for bone diseases were reported. For example, Ignjatović et al ${ }^{67}$ reported a double delivery system where a new nanoparticulate system for controlled and systemic drug delivery with double effect was reported. In their design, the drug is released from the bioresorbable polymer first; then, after resorption of the polymer, nonbioresorbable calcium phosphate remains the chief part of the particle and takes the role of a filler, filling a bone defect.

\section{Nanodelivery of gene therapies for bone diseases}

As our understanding of the pathogenesis of bone diseases deepens, their molecular levels and their molecular mechanisms become clearer to us. Molecule-targeted therapy and gene therapy will become more and more popular. A few studies have reported the development of nanodelivery systems for gene therapy with plasmid DNA. For example, Lu et $\mathrm{l}^{68}$ described the use of hybrid hyaluronic acid (HA)/ CS NPs as gene delivery vectors to transfer exogenous genes into primary chondrocytes for the treatment of joint diseases. In the study, HA/CS plasmid-DNA NPs were synthesized through the complex coacervation of the cationic polymers with a plasmid-expressing enhanced green fluorescent protein $(E G F P)$. Transfection of primary chondrocytes was performed under different conditions to examine transfection efficiency, such as with variations in the $\mathrm{pH}$ of the transfection medium, different $\mathrm{N} / \mathrm{P}$ ratios, different plasmid concentrations, and different molecular weights of CS. They showed that transfection efficiency was at the maximum for a medium $\mathrm{pH}$ of approximately 6.8, an N/P ratio of 5, plasmid concentration of $4 \mu \mathrm{g} / \mathrm{mL}$, and a CS molecular weight of $50 \mathrm{kDa}$. The average viability of cells transfected with HA/ CS-plasmid NPs was over $90 \%$. These results suggest that HA/CS NPs could be an effective nonviral vector for gene delivery to chondrocytes. ${ }^{68}$ However, further testing in vivo is needed to fully assess the effectiveness of this delivery system on gene delivery.

Indeed, an early study using CS as a delivery vehicle to transfer gene expression of $I L-1 R a$ showed a promising result in primary cells. The expression of $I L-1 R a$ was detected in the knee joint synovial fluid of the CS-DNA- (containing IL-1Ra) injected group after direct injection into the knee joint cavities of osteoarthritis rabbits. A significant reduction was noted in the severity of histologic cartilage lesions in the group that received the CS $I L-1 R a$ injection, suggesting that this may represent a promising future treatment for osteoarthritis. ${ }^{60}$ CS-DNA NPs synthesized from the complexation of the cationic polymer with a ss-gal DNA plasmid were also shown to be effective for gene therapy in human mesenchymal stem cells and human osteosarcoma cells (MG63). The researchers showed that transfection of these cells with the NPs resulted in minimal cytotoxicity through specific inhibition of OS cell growth. ${ }^{69}$ These data indicate that CS-based nanomaterials can be used as a potential gene therapy delivery vector for bone diseases. Beside $\mathrm{CS}$, cationic polymers including degraded polyamidoamine dendrimer (SuperFect Transfection Reagent; Qiagen, Hilden, Germany) linear polyethylenimine (ExGen 500; Euromedex, Mundolsheim, Cedex, France), and branched PEI were also reported by Ohashi et $\mathrm{al}^{70}$ for gene delivery into chondrocytes. A plasmid that contains the Escherichia Coli, LacZ (pSES. 
beta) was loaded on to one of above three cationic polymers at different molar ratios and the resultant complex was to transfect a human chondrocyte-like cell line HCS-2/8. Gene expression of $L a c Z$ was measured by an O-nitrophenyl betaD-galactopyranoside (ONPG) assay and by staining with 0.05\% 5-bromo-4-chloro-3-indolyl-beta-D-galactopyranoside. The ONPG assay showed that the highest delivery rate was achieved when $2 \mu \mathrm{g}$ of pSES.beta was combined with either $21 \mu \mathrm{g}$ of dendrimer, $1.7 \mu \mathrm{g}$ of linear PEI, or $2.0 \mu \mathrm{g}$ of branched PEI. LacZ expression reached the highest level 3 days after the dendrimer-mediated transduction, and gradually declined, returning to the background level on day 14 Cytotoxicity or morphological change was not observed at the optimal dose of each polymer, suggesting these cationic polymers, particularly the degraded dendrimer and linear PEI, would be a useful and safe vector for gene delivery to chondrocytes. $^{70}$

Apart from plasmid DNA, RNA interference-based therapy is very popular at this moment for treatments for various diseases including viral infection, genetic disorders, and cancers. ${ }^{71-75}$ However, RNA interference therapy to bone-related diseases (rather than cancer bone metastasis) seems to have just been developed. One example is a report showing the use of diamond NPs (nanodiamonds, NDs) to deliver siRNA into Ewing sarcoma cells. They demonstrated that siRNA was adsorbed onto NDs, which were coated with cationic polymer. Specific inhibition of EWS/Fli-1 gene expression was also observed at the mRNA and protein levels in a serum-containing medium. ${ }^{76,77}$ This promising data warrant a further study about the in vivo system to test its efficiency of tumor treatment.

For Ewing's sarcoma, an early study reported using antisense oligonucleotides (AON) strategy to target the junction area of the fusion gene, EWS-Fli-1, with delivery systems of nanocapsules and NSs. ${ }^{78}$ These nanomaterials were used to deliver a chimeric phosphorothioate, phosphodiester AON, with five additional bases in $5^{\prime}$, which allow this $\mathrm{AON}$ to be structured with a loop. The authors showed that AON against the EWS-Fli-1 oncogene delivered by nanocapsules or NSs inhibited, with high specificity, the growth of an EWS-Fli-1dependent tumor grafted to nude mice. The antisense effect was confirmed by the specific downregulation of EWSFli-1 messenger RNA (mRNA). Conclusions from this study were that both nanocapsules and NSs may be considered as promising systems for AON delivery in vivo. ${ }^{78}$ We believe this direction of research will be intensified soon, and the authors of this review have also carried out some studies using the same strategy to treat osteoarthritis (manuscript is under revision for Nature Communication) and found that the local injection of ERK siRNA delivered by a polymer-based NP can slow down the progress of osteoarthritis. As using NPs to deliver siRNAs to different cells is intensively investigated, these advances will be applied to bone diseases soon. The NPs described above are summarized in Table 2.

\section{The advantages of local delivery in bone tissue}

With the advantages of nanotechnology, it will be possible to achieve localized drug delivery and release in the treatment of bone-related disorders (Figure 2). A few major advantages of local delivery include: (1) retain and kept in local longer therefore increase the treatment time and efficiency; ${ }^{79}$ (2) to reduce the systemic side effects on other cells or organ/ tissues; (3) the dose will be reduced for local application when compared to systemic delivery. The most popularly reported nanomaterial for local delivery is magnetic NPs. These kinds of NPs can be localized in certain bone sites by using a magnetic field; however, the disadvantage of these kinds of NPs is the low specificity to the target cells and the magnetic field may need to be applied for long time.

Pareta et $\mathrm{l}^{80}$ investigated the effect of magnetic NPs on osteoblasts in vitro. It was shown that gamma- $\mathrm{Fe}_{2} \mathrm{O}_{3}$ magnetic NPs could significantly promote osteoblast density (cell number per well) after 5 days and 8 days of culture compared to controls (no particles). The magnetic NPs were also coated with calcium phosphate to tailor them to treat different bone diseases. The coatings were conducted in the presence of either bovine serum albumin or citric acid to reduce magnetic NP agglomeration. Results with these coatings showed that magnetic NPs, specifically (gamma- $\mathrm{Fe}_{2} \mathrm{O}_{3}$ ), coated in the presence of bovine serum albumin significantly increased osteoblast density compared to controls after 1 day. This study provided evidence that calcium phosphate-coated gamma- $\mathrm{Fe}_{2} \mathrm{O}_{3}$ magnetic NPs increased osteoblast density when compared to no particles and, thus, should be further studied to treat numerous bone diseases. ${ }^{80}$

For in vivo cases, a study reported that superparamagnetic iron oxide NPs (SPIONs) co-encapsulated into PLGA microparticles for the purpose of local treatment of inflammatory conditions such as arthritis. The magnetic properties conferred by the SPIONs were shown to help to maintain the microparticles in the joint with an external magnetic field. ${ }^{81}$ The results further showed that the microparticles had an excellent biocompatibility with synoviocytes, and that they were internalized through a phagocytic process, as demonstrated by fluorescence-activated cell sorting and 
Table 2 Summary of NPs used in bone diseases for drug and gene delivery

\begin{tabular}{|c|c|c|c|c|}
\hline Bone diseases & NPs used & Drugs delivered & Drug efficiency & References \\
\hline \multirow[t]{5}{*}{ Cancer bone metastasis } & PLL-CD & BPs (RIS) & Increased & 9 \\
\hline & PLGA & $\mathrm{BPs}(\mathrm{ZOL})$ & Increased & 38 \\
\hline & PLGA & Doxorubicin & Increased & 39 \\
\hline & PLGA & Alendronate & Not detected & 17 \\
\hline & PTX-PEG-ALN & Aminobisphosphonate & Increased & 41 \\
\hline \multirow[t]{10}{*}{ Osteosarcoma and Ewing's sarcoma } & MSN & siRNA & Cell model only & 10 \\
\hline & Polymer & Camptothecin & Increased & 50 \\
\hline & $\mathrm{LDH}$ & Methotrexate & Cell model only & 11 \\
\hline & Chitosan NP & DNA enzyme & Increased & 55 \\
\hline & Chitosan NP & DNA plasmid & Increased & 69 \\
\hline & Polymerized liposomal NP & Doxorubicin & Increased & 45 \\
\hline & Magnetic arsenic trioxide NP & Arsenic trioxide & Increased & 46 \\
\hline & Calcium phosphate NP & Cisplatin & Increased & 43 \\
\hline & $\begin{array}{l}\text { Lipid-modified dextran-based } \\
\text { polymer NP }\end{array}$ & Doxorubicin & Increased & 42 \\
\hline & Dextran-PEI NP & Doxorubicin & Increased & 47 \\
\hline \multirow[t]{2}{*}{ OA } & Polymeric hydrogel & Dextran & Increased & 58 \\
\hline & Chitosan NP & Plasmid DNA & Increased & 60 \\
\hline \multirow[t]{4}{*}{ Infectious and inflammatory } & Porous PLGA & Doxycycline & Increased & 65 \\
\hline & scaffold & & & \\
\hline & mPEG-PLGA hydrogel & Teicoplanin & Increased & 66 \\
\hline & PLGA-calcium phosphate & Tigecycline & Increased & 67 \\
\hline \multirow[t]{4}{*}{ Gene therapy } & HA-chitosan & Plasmid DNA & Model only & 68 \\
\hline & Chitosan & IL-IRa DNA & Increased & 60 \\
\hline & PAMAM dendrimers & LacZ gene & Model study & 70 \\
\hline & Diamond NPs & siRNA to Ewing's sarcoma & Increased & 77 \\
\hline
\end{tabular}

Abbreviations: NP, nanoparticle; PLL-CD, poly-L-lysine covalented beta-cyclodextrin; RIS, risedronate; PLGA, poly lactic-co-glycolic acid; ZOL, zoledronic acid; PTX-PEGALN, poly (ethylene glycol) bearing paclitaxel and alendronate; MSN, mesoporous silica nanoparticle; siRNA, small interfering ribonucleic acid; LDH, layered double hydroxides; DNA, deoxyribonucleic acid; OA, osteoarthritis; mPEG, poly (ethylene glycol) monomethyl ether; HA, hyaluronic acid; PAMAM, polyamidoamine; PEI, polyethylenimine.

morphological analyses of cells exposed to microparticles. Histological examination showed that the microparticles did not induce any inflammatory reactions in the joint, suggesting that this type of carrier could be used as a suitable magnetically retainable intraarticular drug delivery system for treating joint diseases such as arthritis or osteoarthritis. ${ }^{81}$

Apart from magnetic NPs, PLA and PLGA are US Food and Drug Administration (FDA)-approved polymers that are already used for the preparation of nano- or microparticles. HA is a natural polysaccharide that is already present in the articulations known to interact with the CD44 receptors of the cells (especially chondrocytes). Another targeted local delivery system with PLA or PLGA and HA was reported by Zille et al. ${ }^{82}$ In this study, NPs of poly (D, L-lactic acid) (PLA) or PLGA covered by chemically esterified amphiphilic HA were used for intraarticular injection as a drug carrier for the treatment of arthritis and/or osteoarthritis. It was expected that the HA covering could improve the interactions between the cells and the NPs, leading to better targeting or biodistribution. The researchers investigated the cytotoxicity of the NPs. The knees of healthy male rats were injected one to two times weekly with various concentrations of NPs encapsulating dextran-FITC. However, no differences were observed between the control rats and the rats treated with NPs in term of the mRNA expression levels of some specific early cytokines (IL-1beta and tumor necrosis factor-alpha). The results prompted them to test these NPs in osteoarthritis or arthritis rat models, which has not been documented. Another strategy that has not been reported is to use scaffold nanomaterials to load the drug and locally implant the scaffold to the disease site to slowly release the drug.

\section{Nanotechnology in bone regeneration}

Bone defects and malformation, caused by trauma, infection, tumor resection, congenital deformity, as well as physical and pathological degeneration represent a major concern for orthopedic surgeons; nanotechnology plays an important role in bone regeneration. The progress made when using bioactive nanomaterials for bone tissue repair and regeneration has undergone great advances as a result of the scientific efforts aimed at improving the tissue-material response after implantation. Typically, there are two ways to apply nanotechnology to create bioactive nanostructured scaffolds 
to improve bone regeneration. One is to prepare NPs/polymer composite scaffolds; the other is to prepare bioactive glass scaffolds with well ordered nanosized pores.

For the preparation of NPs/polymer composite scaffolds, nanosized HAp, ${ }^{83,84}$ beta-tricalcium phosphate, ${ }^{85,86}$ bioactive glasses, ${ }^{87,88}$ and $\mathrm{CaSiO}_{3}$ particles were mostly incorporated into the polymer matrix. ${ }^{89,90}$ It was found that these bioactive NPs significantly improve the mechanical strength, mineralization ability, degradation, and cytocompatibility of polymer scaffolds. The functionality of nanocomposites is more distinct than that of microsized composites. Therefore, the incorporation of bioactive NPs into biopolymers is a viable way to improve their physiochemical and biological properties for bone regeneration application.

Besides the nanocomposite, the inherent nanostructure is of great importance in improving the bioactivity of bioactive materials. To improve the bioactivity of conventional bioactive glass for bone regeneration, Yan et al, ${ }^{91,92}$ for the first time, prepared a new class of mesoporous bioactive glasses (MBG) in 2004 by the combination of the sol-gel method and supramolecular chemistry of surfactants. Their study has opened a new direction for applying nanotechniques to regenerative medicine by coupling drug delivery with bioactive materials. These materials are based on a CaO- $\mathrm{SiO}_{2}-\mathrm{P}_{2} \mathrm{O}_{5}$ composition and have a highly ordered mesopore channel structure with a pore size ranging from 5-20 nm. Compared to conventional nonmesoporous bioactive glasses, the MBG possesses a more optimal surface area, pore volume, ability to induce in vitro apatite mineralization in simulated body fluids, and excellent cytocompatibility. ${ }^{92-95}$ For better bone regeneration application, $\mathrm{MBG}$ can also be prepared as 3D porous scaffolds for bone tissue engineering and drug delivery applications. ${ }^{96}$ Currently, there are three methods to prepare MBG scaffolds. The first MBG scaffold was prepared by the porogen method. Yun et $\mathrm{al}^{97}$ applied methyl cellulose as the porogen to prepare porous MBG scaffolds with a large-pore size of $100 \mu \mathrm{m}$. The second scaffold was prepared by the polymer template method, which is widely used. We have developed a series of MBG scaffolds with varying compositions for drug delivery and bone tissue engineering application. ${ }^{98-101}$ The prepared scaffolds possess large pores with the size of $300-500 \mu \mathrm{m}$, and well-ordered mesopores with the pore size of $5 \mathrm{~nm}$ (Figure 3 ). The advantages of the MBG scaffolds prepared by polyurethane sponge template method include their highly interconnective pore structures and controllable pore size (porosity), while the disadvantage is the low mechanical strength of the material. ${ }^{102}$

To better control the pore morphology, pore size, and porosity, a 3D plotting technique (also called direct writing or printing) has been developed to prepare porous $\mathrm{MBG}$ scaffolds. The significant advantage of this technique is that the architectures of the scaffolds can be concisely controlled by layer-by-layer plotting under mild conditions. ${ }^{103-105}$ Recently, a new facile method was used to prepare hierarchical and multifunctional MBG scaffolds with controllable pore architecture, excellent mechanical strength, and mineralization ability for bone regeneration application by a modified 3D-printing technique using polyvinylalcohol as a binder. The obtained 3D-printing MBG scaffolds possess a high mechanical strength, which is about 200 times that of the MBG scaffolds prepared using traditional polyurethane foam as templates. They have highly controllable pore architecture and excellent apatite-mineralization ability, as well as a sustained drug-delivery property. ${ }^{96,106}$

MBG scaffolds could efficiently deliver drug and growth factors. Dexamethasone (DEX) was loaded into MBG scaf-
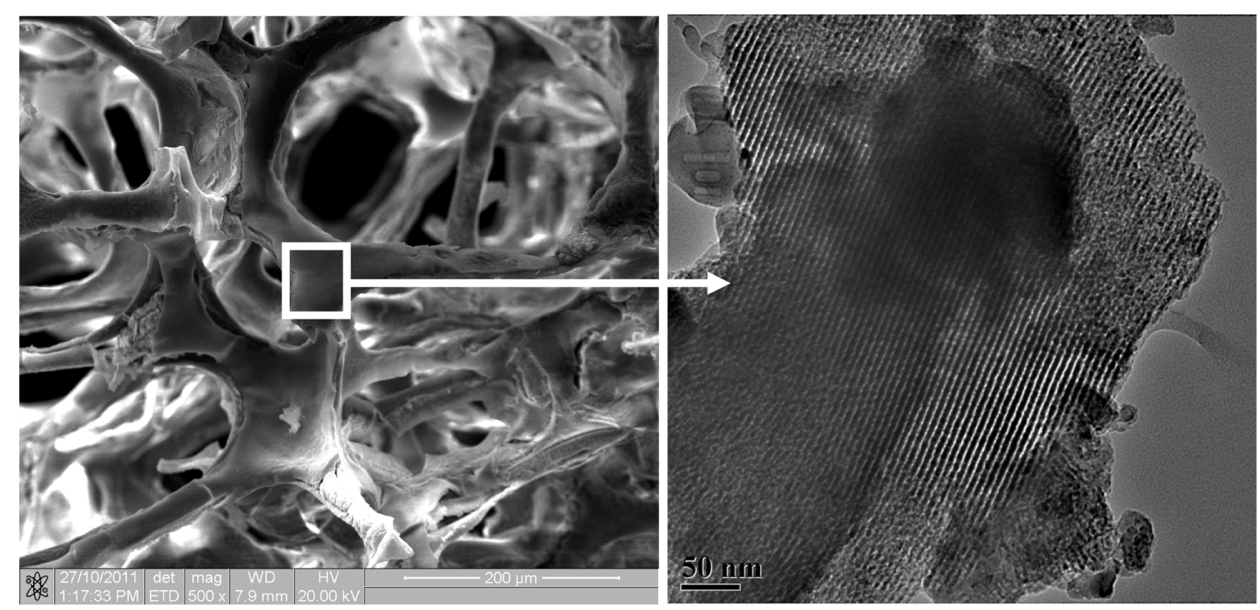

Figure 3 Porous mesoporous bioactive glass scaffolds with large pores (several hundred micrometers, left) and well-ordered mesoporous channel structures (5 nm, right). 
folds, and it was found that the sustained release of DEX from MBG scaffolds significantly enhanced alkaline phosphatase (ALP) activity and gene expressions (ALP, bone sialoprotein and Col I) of osteoblasts. ${ }^{96}$ These results suggest that DEXloaded MBG scaffolds show great potential as a release system to enhance osteogenesis, and may be used for bone tissue engineering application. ${ }^{96,100}$ The effect of VEGF delivery from MBG scaffolds on the viability of endothelial cells was further investigated and it was found that the mesopore structures in MBG scaffolds play an important role in maintaining the bioactivity of VEGF, further improving the viability of endothelial cells, indicating that MBG scaffolds are an excellent carrier of VEGF for stimulating angiogenesis. ${ }^{107}$ Therefore, MBG scaffolds, as a typical nanobiomaterial, combine the drug delivery and bioactivity for better bone regeneration application by harnessing their unique nanopore structure. The combination of drug delivery and bioactivity may be a new concept for tissue regeneration by the functional effect of nanomaterials.

\section{Conclusion and future perspective}

Nanotechnology has shown a bright future in treating bone diseases, as evidenced by some promising results in in vitro or in vivo studies. However, in vivo validation of these reported nanomaterials, and particularly subsequent toxicity testing and bone tissue targeted delivery for either cancer bone metastasis or other bone diseases, still need further and deep studies to facilitate their future clinical application. Some nanomaterials such as LDH, which has the same composition as FDAapproved alum adjuvant will be ready to use in humans. Some other polymer-based NPs like CS and PLGA do not have much cytotoxicity, and may also be expected to be applied to humans in the near future. Calcium phosphate-based NPs have also been used in drug delivery for bone diseases and are not supposed to be toxic to bone tissues. Thus, these nanomaterials will certainly be the focus of future research and clinical applications. It is expected that nanotechnology will play more important roles in the future treatments of bone diseases and bone regeneration. Local delivery systems and multifunctional NPs with targeted delivery specific to bone tissues or cells will soon be seen, with better controlled release and escape from endosomes, if drug delivery needs to occur in the cytoplasm (such as siRNA). More effective treatments including the big improvement of current therapies for bone diseases will be seen with the advancement of the technology in the near future.

\section{Acknowledgments}

The authors would like to acknowledge the funding support from the National Health and Medical Research Council
APP1032738 and the Australia Research Council DP 120103697.

\section{Disclosure}

The authors report no conflicts of interest in this work.

\section{References}

1. Giljohann DA, Mirkin CA. Drivers of biodiagnostic development. Nature. 2009;462(7272):461-464.

2. Cheng Z, Al Zaki A, Hui JZ, Muzykantov VR, Tsourkas A. Multifunctional nanoparticles: cost versus benefit of adding targeting and imaging capabilities. Science. 2012;338(6109):903-910.

3. Colson YL, Grinstaff MW. Biologically responsive polymeric nanoparticles for drug delivery. Adv Mater. 2012;24(28):3878-3886.

4. Tang F, Li L, Chen D. Mesoporous silica nanoparticles: synthesis, biocompatibility and drug delivery. Adv Mater. 2012;24(12): 1504-1534.

5. Danhier F, Ansorena E, Silva JM, Coco R, Le Breton A, Préat V. PLGA-based nanoparticles: an overview of biomedical applications. J Control Release. 2012;161(2):505-522.

6. Papasani MR, Wang G, Hill RA. Gold nanoparticles: the importance of physiological principles to devise strategies for targeted drug delivery. Nanomedicine. 2012;8(6):804-814.

7. Dvir T, Timko BP, Kohane DS, Langer R. Nanotechnological strategies for engineering complex tissues. Nat Nanotechnol. 2011;6(1):13-22.

8. Elazar V, Adwan H, Bäuerle T, Rohekar K, Golomb G, Berger MR. Sustained delivery and efficacy of polymeric nanoparticles containing osteopontin and bone sialoprotein antisenses in rats with breast cancer bone metastasis. Int J Cancer. 2010;126(7):1749-1760.

9. Daubiné F, Cortial D, Ladam G, et al. Nanostructured polyelectrolyte multilayer drug delivery systems for bone metastasis prevention. Biomaterials. 2009;30(31):6367-6373.

10. Hartono SB, Gu W, Kleitz F, et al. Poly-L-lysine functionalized large pore cubic mesostructured silica nanoparticles as biocompatible carriers for gene delivery. ACS Nano. 2012;6(3):2104-2117.

11. Oh JM, Park CB, Choy JH. Intracellular drug delivery of layered double hydroxide nanoparticles. J Nanosci Nanotechnol. 2011;11(2): 1632-1635.

12. Clézardin P, Benzaïd I, Croucher PI. Bisphosphonates in preclinical bone oncology. Bone. 2011;49(1):66-70.

13. Coleman R. The use of bisphosphonates in cancer treatment. Ann NY Acad Sci. 2011;1218:3-14.

14. Diel IJ, Solomayer EF, Costa SD, et al. Reduction in new metastases in breast cancer with adjuvant clodronate treatment. $N$ Engl J Med. 1998; 339(6):357-363.

15. Lipton A. Emerging role of bisphosphonates in the clinic - antitumor activity and prevention of metastasis to bone. Cancer Treat Rev. 2008; 34 Suppl 1:S25-S30.

16. Sun M, Igbal J, Singh S, Sun L, Zaidi M. The crossover of bisphosphonates to cancer therapy. Ann NY Acad Sci. 2010;1211:107-112.

17. Pignatello R, Cenni E, Micieli D, et al. A novel biomaterial for osteotropic drug nanocarriers: synthesis and biocompatibility evaluation of a PLGA-ALE conjugate. Nanomedicine (Lond). 2009;4(2): 161-175.

18. Luo B, Song XJ, Zhang F, et al. Multi-functional thermosensitive composite microspheres with high magnetic susceptibility based on magnetite colloidal nanoparticle clusters. Langmuir. 2010;26(3): $1674-1679$

19. Jabr-Milane L, van Vlerken L, Devalapally H, et al. Multi-functional nanocarriers for targeted delivery of drugs and genes. J Control Release. 2008;130(2):121-128.

20. Wu W, Shen J, Gai Z, Hong K, Banerjee P, Zhou S. Multi-functional core-shell hybrid nanogels for $\mathrm{pH}$-dependent magnetic manipulation, fluorescent pH-sensing, and drug delivery. Biomaterials. 2011;32(36): 9876-9887. 
21. Xu H, Cheng L, Wang C, Ma X, Li Y, Liu Z. Polymer encapsulated upconversion nanoparticle/iron oxide nanocomposites for multimodal imaging and magnetic targeted drug delivery. Biomaterials. 2011;32(35):9364-9373.

22. Yallapu MM, Othman SF, Curtis ET, et al. Multi-functional magnetic nanoparticles for magnetic resonance imaging and cancer therapy. Biomaterials. 2011;32(7):1890-1905.

23. Jimenez-Andrade JM, Mantyh WG, Bloom AP, Ferng AS, Geffre CP, Mantyh PW. Bone cancer pain. Ann NY Acad Sci. 2010;1198: 173-181.

24. Guise T. Examining the metastatic niche: targeting the microenvironment. Semin Oncol. 2010;37 Suppl 2:S2-S14.

25. Coleman RE. Clinical features of metastatic bone disease and risk of skeletal morbidity. Clin Cancer Res. 2006;12(20 Pt 2):6243s-6249s.

26. Russell RG, Rogers MJ. Bisphosphonates: from the laboratory to the clinic and back again. Bone. 1999;25(1):97-106.

27. Lipton A. New therapeutic agents for the treatment of bone diseases Expert Opin Biol Ther. 2005;5(6):817-832.

28. Roelofs AJ, Thompson K, Gordon S, Rogers MJ. Molecular mechanisms of action of bisphosphonates: current status. Clin Cancer Res 2006;12(20 Pt 2):6222s-6230s.

29. Coleman RE, McCloskey EV. Bisphosphonates in oncology. Bone. 2011;49(1):71-76

30. Gnant M. Bisphosphonates in the prevention of disease recurrence: current results and ongoing trials. Curr Cancer Drug Targets. 2009;9(7): 824-833.

31. Hurst M, Noble S. Clodronate: a review of its use in breast cancer. Drugs Aging. 1999;15(2):143-167.

32. Panasci LC, Sandor V, Melnychuk D. Clodronate in metastatic breas cancer. $N$ Engl J Med. 1998;339(26):1940-1941; author reply 1941.

33. Paterson AH, Anderson SJ, Lembersky BC, et al. Oral clodronate for adjuvant treatment of operable breast cancer (National Surgical Adjuvant Breast and Bowel Project protocol B-34): a multicentre, placebocontrolled, randomised trial. Lancet Oncol. 2012;13(7):734-742.

34. Santini D, Vincenzi B, Hannon RA, et al. Changes in bone resorption and vascular endothelial growth factor after a single zoledronic acid infusion in cancer patients with bone metastases from solid tumours. Oncol Rep. 2006;15(5):1351-1357.

35. Zhao X, Xu X, Guo L, et al. Biomarker alterations with metronomic use of low-dose zoledronic acid for breast cancer patients with bone metastases and potential clinical significance. Breast Cancer Res Treat. 2010;124(3):733-743.

36. Meulenbeld HJ, van Werkhoven ED, Coenen JL, et al. Randomised phase II/III study of docetaxel with or without risedronate in patients with metastatic Castration Resistant Prostate Cancer (CRPC), The Netherlands Prostate Study (NePro). Eur J Cancer. 2012;48(16): 2993-3000.

37. Kim SW, Kim JS, Papadopoulos J, et al. Consistent interactions between tumor cell IL- 6 and macrophage TNF- $\alpha$ enhance the growth of human prostate cancer cells in the bone of nude mouse. Int Immunopharmacol. 2011;11(7):862-872

38. Ramanlal Chaudhari K, Kumar A, Megraj Khandewlwal VK, et al. Bone metastasis targeting: a novel approach to reach bone using Zoledronate anchored PLGA nanoparticle as carrier system loaded with Docetaxel. J Control Release. 2012;158(3):470-478.

39. Salerno M, Cenni E, Fotia C, et al. Bone-targeted doxorubicin-loaded nanoparticles as a tool for the treatment of skeletal metastases. Curr Cancer Drug Targets. 2010;10(7):649-659.

40. Pillai RR, Somayaji SN, Rabinovich M, Hudson MC, Gonsalves KE Nafcillin-loaded PLGA nanoparticles for treatment of osteomyelitis. Biomed Mater. 2008;3(3):034114.

41. Clementi C, Miller K, Mero A, Satchi-Fainaro R, Pasut G. Dendritic poly(ethylene glycol) bearing paclitaxel and alendronate for targeting bone neoplasms. Mol Pharm. 2011;8(4):1063-1072.

42. Susa M, Iyer AK, Hornicek FJ, Mankin H, Amiji MM, Duan Z. Doxorubicin loaded polymeric nanoparticulate delivery system to overcome drug resistance in osteosarcoma. BMC Cancer. 2009;9:399.
43. Barroug A, Kuhn LT, Gerstenfeld LC, Glimcher MJ. Interactions of cisplatin with calcium phosphate nanoparticles: in vitro controlled adsorption and release. J Orthop Res. 2004;22(4):703-708.

44. Reufsteck C, Lifshitz-Shovali R, Zepp M, et al. Silencing of skeletal metastasis-associated genes impairs migration of breast cancer cells and reduces osteolytic bone lesions. Clin Exp Metastasis. 2012;29(5): $441-456$.

45. Federman N, Chan J, Nagy JO, et al. Enhanced growth inhibition of osteosarcoma by cytotoxic polymerized liposomal nanoparticles targeting the alcam cell surface receptor. Sarcoma. 2012;2012:126906.

46. Li XS, Li WQ, Wang WB. Using targeted magnetic arsenic trioxide nanoparticles for osteosarcoma treatment. Cancer Biother Radiopharm. 2007;22(6):772-778.

47. Sun K, Wang J, Zhang J, Hua M, Liu C, Chen T. Dextran-g-PEI nanoparticles as a carrier for co-delivery of adriamycin and plasmid into osteosarcoma cells. Int J Biol Macromol. 2011;49(2):173-180.

48. Mandal D, Maran A, Yaszemski MJ, Bolander ME, Sarkar G. Cellular uptake of gold nanoparticles directly cross-linked with carrier peptides by osteosarcoma cells. J Mater Sci Mater Med. 2009;20(1):347-350.

49. Cebrián V, Martín-Saavedra F, Yagüe C, Arruebo M, Santamaría J, Vilaboa N. Size-dependent transfection efficiency of PEI-coated gold nanoparticles. Acta Biomater. 2011;7(10):3645-3655.

50. Schluep T, Hwang J, Cheng J, et al. Preclinical efficacy of the camptothecin-polymer conjugate IT-101 in multiple cancer models. Clin Cancer Res. 2006;12(5):1606-1614.

51. Susa M, Iyer AK, Ryu K, et al. Inhibition of ABCB1 (MDR1) expression by an siRNA nanoparticulate delivery system to overcome drug resistance in osteosarcoma. PLoS One. 2010;5(5):e10764.

52. Andersen MØ, Lichawska A, Arpanaei A, et al. Surface functionalisation of PLGA nanoparticles for gene silencing. Biomaterials. 2010; 31(21):5671-5677.

53. Kaul Z, Yaguchi T, Kaul SC, Wadhwa R. Quantum dot-based protein imaging and functional significance of two mitochondrial chaperones in cellular senescence and carcinogenesis. Ann N Y Acad Sci. 2006; 1067:469-473.

54. Wang GW, Guo W, Tang XD, Peng CL, Zhao H. Lethal effects of nanoliposome encapsulated cisplatin on Saos-2 cells and its distribution in nude mice bearing human. Beijing Da Xue Xue Bao. 2009; 41(5):525-530. Chinese.

55. Tan ML, Dunstan DE, Friedhuber AM, Choong PF, Dass CR. A nanoparticulate system that enhances the efficacy of the tumoricide Dz13 when administered proximal to the lesion site. J Control Release. 2010;144(2):196-202.

56. Li Y, Lim S, Ooi CP. Fabrication of cisplatin-loaded poly(lactide-coglycolide) composite microspheres for osteosarcoma treatment. Pharm Res. 2012;29(3):756-769.

57. Azarmi S, Huang Y, Chen H, et al. Optimization of a two-step desolvation method for preparing gelatin nanoparticles and cell uptake studies in 143B osteosarcoma cancer cells. J Pharm Pharm Sci. 2006; 9(1):124-132.

58. Morgen M, Tung D, Boras B, Miller W, Malfait AM, Tortorella M. Nanoparticles for improved local retention after intra-articular injection into the knee joint. Pharm Res. 2013;30(1):257-268.

59. Whitmire RE, Wilson DS, Singh A, Levenston ME, Murthy N, García AJ. Self-assembling nanoparticles for intra-articular delivery of anti-inflammatory proteins. Biomaterials. 2012;33(30):7665-7675.

60. Zhang X, Yu C, Xushi, Zhang C, Tang T, Dai K. Direct chitosanmediated gene delivery to the rabbit knee joints in vitro and in vivo. Biochem Biophys Res Commun. 2006;341(1):202-208.

61. Simon GH, von Vopelius-Feldt J, Fu Y, et al. Ultrasmall supraparamagnetic iron oxide-enhanced magnetic resonance imaging of antigen-induced arthritis: a comparative study between SHU 555 C, ferumoxtran-10, and ferumoxytol. Invest Radiol. 2006;41(1):45-51.

62. Chiang CY, Hsieh ML, Huang KW, Chau LK, Chang CM, Lyu SR. Fiber-optic particle plasmon resonance sensor for detection of interleukin-1 $\beta$ in synovial fluids. Biosens Bioelectron. 2010;26(3): 1036-1042. 
63. Baker BM, Mauck RL. The effect of nanofiber alignment on the maturation of engineered meniscus constructs. Biomaterials. 2007; 28(11):1967-1977.

64. Yang L, Webster TJ. Nanotechnology controlled drug delivery for treating bone diseases. Expert Opin Drug Deliv. 2009;6(8):851-864.

65. Feng K, Sun H, Bradley MA, Dupler EJ, Giannobile WV, Ma PX. Novel antibacterial nanofibrous PLLA scaffolds. J Control Release. 2010;146(3):363-369.

66. Peng KT, Chen CF, Chu IM, et al. Treatment of osteomyelitis with teicoplanin-encapsulated biodegradable thermosensitive hydrogel nanoparticles. Biomaterials. 2010;31(19):5227-5236.

67. Ignjatović NL, Ninkov P, Sabetrasekh R, Uskoković DP. A novel nano drug delivery system based on tigecycline-loaded calciumphosphate coated with poly-DL-lactide-co-glycolide. J Mater Sci Mater Med. 2010;21(1):231-239.

68. Lu HD, Zhao HQ, Wang K, Lv LL. Novel hyaluronic acid-chitosan nanoparticles as non-viral gene delivery vectors targeting osteoarthritis. Int J Pharm. 2011;420(2):358-365.

69. Corsi K, Chellat F, Yahia L, Fernandes JC. Mesenchymal stem cells, MG63 and HEK293 transfection using chitosan-DNA nanoparticles. Biomaterials. 2003;24(7):1255-1264.

70. Ohashi S, Kubo T, Ikeda T, et al. Cationic polymer-mediated genetic transduction into cultured human chondrosarcoma-derived HCS2/8 cells. J Orthop Sci. 2001;6(1):75-81.

71. Harper SQ, Staber PD, He X, et al. RNA interference improves motor and neuropathological abnormalities in a Huntington's disease mouse model. Proc Natl Acad Sci U S A. 2005;102(16):5820-5825.

72. Qin XF, An DS, Chen IS, Baltimore D. Inhibiting HIV-1 infection in human T cells by lentiviral-mediated delivery of small interfering RNA against CCR5. Proc Natl Acad Sci U S A. 2003;100(1):183-188.

73. Jacque JM, Triques K, Stevenson M. Modulation of HIV-1 replication by RNA interference. Nature. 2002;418(6896):435-438.

74. Gu W, Cochrane M, Leggatt GR, et al. Both treated and untreated tumors are eliminated by short hairpin RNA-based induction of targetspecific immune responses. Proc Natl Acad Sci U S A. 2009;106(20): 8314-8319

75. Gu W, Putral LN, Irving A, McMillan NA. The development and future of oligonucleotide-based therapies for cervical cancer. Curr Opin Mol Ther. 2007;9(2):126-131.

76. Toub N, Bertrand JR, Tamaddon A, et al. Efficacy of siRNA nanocapsules targeted against the EWS-Flil oncogene in Ewing sarcoma. Pharm Res. 2006;23(5):892-900.

77. Alhaddad A, Adam MP, Botsoa J, et al. Nanodiamond as a vector for siRNA delivery to Ewing sarcoma cells. Small. 2011;7(21):3087-3095.

78. Maksimenko A, Malvy C, Lambert G, et al. Oligonucleotides targeted against a junction oncogene are made efficient by nanotechnologies. Pharm Res. 2003;20(10):1565-1567.

79. Wang L, Cao J, Lei DL, et al. Application of nerve growth factor by gel increases formation of bone in mandibular distraction osteogenesis in rabbits. Br J Oral Maxillofac Surg. 2010;48(7):515-519.

80. Pareta RA, Taylor E, Webster TJ. Increased osteoblast density in the presence of novel calcium phosphate coated magnetic nanoparticles. Nanotechnology. 2008;19(26):265101.

81. Butoescu N, Seemayer CA, Foti M, Jordan O, Doelker E. Dexamethasone-containing PLGA superparamagnetic microparticles as carriers for the local treatment of arthritis. Biomaterials. 2009;30(9): 1772-1780.

82. Zille H, Paquet J, Henrionnet C, et al. Evaluation of intra-articular delivery of hyaluronic acid functionalized biopolymeric nanoparticles in healthy rat knees. Biomed Mater Eng. 2010;20(3):235-242.

83. Abdal-hay A, Sheikh FA, Lim JK. Air jet spinning of hydroxyapatite/ poly(lactic acid) hybrid nanocomposite membrane mats for bone tissue engineering. Colloids Surf B Biointerfaces. 2012;102:635-643.

84. Im O, Li J, Wang M, Zhang LG, Keidar M. Biomimetic threedimensional nanocrystalline hydroxyapatite and magnetically synthesized single-walled carbon nanotube chitosan nanocomposite for bone regeneration. Int J Nanomedicine. 2012;7:2087-2099.
85. Kim J, McBride S, Tellis B, et al. Rapid-prototyped PLGA/ $\beta-T C P /$ hydroxyapatite nanocomposite scaffolds in a rabbit femoral defect model. Biofabrication. 2012;4(2):025003.

86. Wilberforce SI, Finlayson CE, Best SM, Cameron RE. A comparative study of the thermal and dynamic mechanical behaviour of quenched and annealed bioresorbable poly-L-lactide/ $\alpha$-tricalcium phosphate nanocomposites. Acta Biomater. 2012;7(5):2176-2184.

87. Marelli B, Ghezzi CE, Mohn D, et al. Accelerated mineralization of dense collagen-nano bioactive glass hybrid gels increases scaffold stiffness and regulates osteoblastic function. Biomaterials. 2011;32(34): 8915-8926.

88. Jo JH, Lee EJ, Shin DS, et al. In vitro/in vivo biocompatibility and mechanical properties of bioactive glass nanofiber and poly(epsiloncaprolactone) composite materials. J Biomed Mater Res B Appl Biomater. 2009;91(1):213-220.

89. Wei J, Heo SJ, Liu C, et al. Preparation and characterization of bioactive calcium silicate and poly(epsilon-caprolactone) nanocomposite for bone tissue regeneration. J Biomed Mater Res A. 2009; 90(3):702-712.

90. Dou Y, Wu C, Chang J. Preparation, mechanical property and cytocompatibility of poly(L-lactic acid)/calcium silicate nanocomposites with controllable distribution of calcium silicate nanowires. Acta Biomater. 2012;8(11):4139-4150.

91. Yan X, Yu C, Zhou X, Tang J, Zhao D. Highly ordered mesoporous bioactive glasses with superior in vitro bone-forming bioactivities. Angew Chem Int Ed Engl. 2004;43(44):5980-5984.

92. Yan X, Huang X, Yu C, et al. The in-vitro bioactivity of mesoporous bioactive glasses. Biomaterials. 2006;27(18):3396-3403.

93. Leonova E, Izquierdo-Barba I, Arcos D, et al. Multinuclear solid-state NMR studies of ordered mesoporous bioactive glasses. J Phys Chem C Nanomater Interfaces. 2008;112(14):5552-5562.

94. García A, Cicuéndez M, Izquierdo-Barba I, Arcos D, Vallet-Regi M. Essential role of calcium phosphate heterogeneities in 2D-hexagonal and 3D-cubic SiO2-CaO-P2O5 mesoporous bioactive glasses. Chem Mater. 2009;21(22):5474-5484.

95. Alcaide M, Portolés P, López-Noriega A, Arcos D, Vallet-Regí M, Portolés MT. Interaction of an ordered mesoporous bioactive glass with osteoblasts, fibroblasts and lymphocytes, demonstrating its biocompatibility as a potential bone graft material. Acta Biomater. 2010; 6(3):892-899.

96. Wu C, Chang J. Mesoporous bioactive glasses: structure characteristics, drug/growth factor delivery and bone regeneration application. Interface Focus. 2012;2(3):292-306.

97. Yun HS, Kim SE, Hyun YT, Heo SJ, Shin JW. Hierarchically mesoporous-macroporous bioactive glasses scaffolds for bone tissue regeneration. J Biomed Mater Res B Appl Biomater. 2008;87(2): 374-380.

98. Wu C, Fan W, Gelinsky M, et al. Bioactive SrO-SiO(2) glass with wellordered mesopores: characterization, physiochemistry and biological properties. Acta Biomater. 2011;7(4):1797-1806.

99. Wu C, Fan W, Zhu Y, et al. Multifunctional magnetic mesoporous bioactive glass scaffolds with a hierarchical pore structure. Acta Biomater. 2011;7(10):3563-3572.

100. Wu C, Miron R, Sculean A, et al. Proliferation, differentiation and gene expression of osteoblasts in boron-containing associated with dexamethasone deliver from mesoporous bioactive glass scaffolds. Biomaterials. 2011;32(29):7068-7078.

101. Zhu Y, Zhang Y, Wu C, Fang Y, Yang J, Wang S. The effect of zirconium incorporation on the physiochemical and biological properties of mesoporous bioactive glasses scaffolds. Microporous and Mesoporous Materials. 2011;143(2-3):311-319.

102. Wu C, Zhang Y, Zhu Y, Friis T, Xiao Y. Structure-property relationships of silk-modified mesoporous bioglass scaffolds. Biomaterials. 2010;31(13):3429-3438.

103. Franco J, Hunger P, Launey ME, Tomsia AP, Saiz E. Direct write assembly of calcium phosphate scaffolds using a water-based hydrogel. Acta Biomater. 2010;6(1):218-228. 
104. Miranda P, Pajares A, Saiz E, Tomsia AP, Guiberteau F. Mechanical properties of calcium phosphate scaffolds fabricated by robocasting. J Biomed Mater Res A. 2008;85(1):218-227.

105. Miranda P, Saiz E, Gryn K, Tomsia AP. Sintering and robocasting of beta-tricalcium phosphate scaffolds for orthopaedic applications. Acta Biomater. 2006;2(4):457-466.

106. Wu C, Luo Y, Cuniberti G, Xiao Y, Gelinsky M. Three-dimensional printing of hierarchical and tough mesoporous bioactive glass scaffolds with a controllable pore architecture, excellent mechanical strength and mineralization ability. Acta Biomater. 2011;7(6):2644-2650.
107. Wu C, Fan W, Chang J, Xiao Y. Mesoporous bioactive glass scaffolds for efficient delivery of vascular endothelial growth factor. J Biomater Appl. Epub July 10, 2012.

International Journal of Nanomedicine

\section{Publish your work in this journal}

The International Journal of Nanomedicine is an international, peerreviewed journal focusing on the application of nanotechnology in diagnostics, therapeutics, and drug delivery systems throughout the biomedical field. This journal is indexed on PubMed Central,

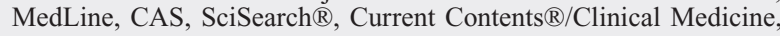

Journal Citation Reports/Science Edition, EMBase, Scopus and the Elsevier Bibliographic databases. The manuscript management system is completely online and includes a very quick and fair peer-review system, which is all easy to use. Visit http://www.dovepress.com/ testimonials.php to read real quotes from published authors.

Submit your manuscript here: http://www.dovepress.com/international-journal-of-nanomedicine-journal 